\title{
Effects of Local Thermotherapy on Chest Pain in Patients with Acute Coronary Syndrome: A Clinical Trial
}

\author{
Amin Moradkhani ${ }^{1}$, Shahram Baraz (iD ${ }^{1,}{ }^{*}$, Habib Haybar $^{2}$, Akram Hemmatipour $^{3}$ and Saeed Hesam ${ }^{4}$ \\ ${ }^{1}$ Nursing Care Research Center in Chronic Diseases, School of Nursing and Midwifery, Ahvaz Jundishapur University of Medical Sciences, Ahvaz, Iran \\ ${ }^{2}$ Atherosclerosis Research Center, Ahvaz Jundishapur University of Medical Sciences, Ahvaz, Iran \\ ${ }^{3}$ Shoushtar University of Medical Sciences, Shoushtar, Iran \\ ${ }^{4}$ Department of Epidemiology and Biostatistics, School of Public Health, Tehran University of Medical Sciences, Tehran, Iran \\ "Corresponding author: Nursing Care Research Center in Chronic Diseases, School of Nursing and Midwifery, Ahvaz Jundishapur University of Medical Sciences, Ahvaz, Iran. \\ Email: shahrambaraz@ajums.ac.ir
}

Received 2018 April 24; Revised 2018 September 25; Accepted 2018 October 15.

\begin{abstract}
Background: The chest pain is a very common complaint among patients with acute coronary syndrome (ACS). The local thermotherapy can reduce or relieve the heart pain by suppressing metabolites as pain mediators.

Objectives: This study aimed to determine the effect of local thermotherapy on the chest pain in patients with ACS.

Methods: This clinical trial was conducted on 78 patients with ACS hospitalized in the ICU of Golestan Hospital of Ahvaz in 2016. Based on the sample size and inclusion criteria, and by a convenience sampling, the eligible patients were enrolled and randomized into two equal-sized groups, namely control and intervention, each containing 39 participants. During the local thermotherapy sessions, the patients received local heat by using a hot pack warmed to $50^{\circ} \mathrm{C}$ for five days. The control group only received the routine treatment. Data collection instruments included a demographic questionnaire and the Numerical Rating Scale (NRS). The NRS was completed by the participants before the intervention and five days after it. Data were analyzed using the descriptive tests, correlation coefficient, independent $t$-test, Chi-square, and logistic regression in SPSS 20.

Results: The mean pain severity in the case group before the intervention was $3.22 \pm 0.86$ and after the intervention, it decreased to $2.61 \pm 0.7$, indicating the effect of local thermotherapy. However, it was not statistically significant $(\mathrm{P}>0.05)$. There was no significant relationship between age, gender, diabetes, hypertension, and hyperlipidemia, and the pain severity in this study (P $>$ 0.05). Logistic regression analysis showed that women experienced more pain than men did.

Conclusions: This study suggested the slight effectiveness of local thermotherapy in reducing the severity of pain in these patients. As a result, further studies are recommended.
\end{abstract}

Keywords: Local Thermotherapy, ACS, Hot Pack

\section{Background}

A significant number of hospitalized patients at hospitals are patients with heart disease, especially patients with acute coronary syndrome (ACS) (1). The ACS is one of the manifestations of coronary artery disease, which results in the death of the cell as a result of the disparity between the intake of oxygen and the required oxygen due to reduced coronary blood flow (2,3). Approximately, 1.1 million patients are admitted to hospitals each year in the United States for the diagnosis of ACS, of which more than onethird are women (3). The syndrome represents a range of early-onset deaths (30 days) ranging from one to $10 \%$ and recurrence rates of 5\% - 15\% during the first year (4-6). In Iran, the ACS is the most common cause of death, accounting for about $46 \%$ of the deaths (5).
The aim of pain treatment in patients with ACS is to restore myocardial blood flow, prevent or minimize cardiac damage, and prevent heart rate. However, increased blood pressure and the myocardium need for oxygen are some of the psychosocial problems of patients due to hospitalization (7-9).

Since the pain relief is the core of nursing care, the importance of playing this role by nurses who have a key role in pain management is more highlighted $(10,11)$. In addition, nurses spend time more than other medical staff with patients and they always decide on pain evaluation and management, specifically in ICUs (12).

Medicines such as morphine and nonpharmacological drugs are used to control the pain of coronary patients. Due to adverse drug reactions and differences in patient response, it is important that non- 
pharmacological methods along with analgesics be used to reduce pain and anxiety in patients because of their fewer complications and more access and effectiveness (7, 13).

Today, medical science tries to reduce medicine consumption or replace it by non-medicinal methods. This is because non-pharmacological techniques are associated with fewer side effects, and are more effective and accessible. Thermotherapy is a non-medicinal pain management technique, which can improve the signs and symptoms of these patients through different mechanisms (14).

Today, thermotherapy can act in two different ways for pain management: either on the surface tissues (skin) or in deep tissues (muscles) (15). Thermotherapy improves the symptoms by disposing the toxic metabolites, such as histamine and bradykinin, from the affected region, vasodilation, increasing blood flow to the inflamed and damaged area, and reducing sympathetic activity $(14,16)$. In a study, Nadler et al. showed the greater effectiveness of thermotherapy compared to ibuprofen and acetaminophen pills in relieving back pain (17).

There are several methods for delivering thermotherapy to the body, including hot packs. These packs are specifically heated pouches that in addition to delivery of heat and increase of blood flow in the region, allow the heat to penetrate into deeper tissues (18). In a study, Uchiyama-Tanaka showed that the application of hot packs in hemodialysis patients with acute cardiac disease reduced brain natriuretic peptide and normalized perfusion (19). In a study conducted by Mohammadian et al., the use of hot water pouch locally in the posterior part of the chest of patients with ACS was associated with decreased pain (5). Takayama et al. (20), Yildirim et al. (21), Yaghobi (18), Behmanesh (22), and Mohammadian (5) also reported the effectiveness of local thermotherapy in relieving pain in patients.

In contrast, Cui and Sinoway reported the negative effect of thermal stress on sympathetic nerves by increasing their activity in patients with chronic heart failure (23).

Regarding the condition of patients with ACS, the use of the sauna and thermotherapy in the acute stage of the disease is not possible (7). The symptoms may improve with local thermotherapy using hot packs, and the effects may be similar to the effects of a whole-body thermotherapy.

\section{Objectives}

Regarding the scant studies of local thermotherapy and its advantages (e.g. improvement of circulation, reduction of pain, improvement of physiological indices, universal accessibility, and ease of use), we aimed to determine the effect of local thermotherapy on the chest pain in patients with ACS hospitalized at Golestan Hospital in Ahvaz in 2015.

\section{Methods}

This clinical trial with code IRCT20161122031017N2 was registered in 2017. The statistical population included all patients with ACS hospitalized at the ICU of Ahvaz Golestan Hospital.

78 patients were selected using a purposive sampling strategy based on the difference between the means, the randomized complete block design with four factors, and inclusion criteria. The participants were then equally placed in the intervention or control groups.

The inclusion criteria were patients aged over 30-yearsold, without addiction, without a history of psychological, muscle, and gastrointestinal problems, without a decreased level of consciousness, and without chest wound and scar.

The exclusion criteria were an unwillingness to participate and an incomplete questionnaire.

Data collection instruments included:

1. Demographic questionnaire (age, gender, history of diabetes, hypertension, and hyperlipidemia)

2. Numerical Rating Scale (NRS)

In the Numerical Rating Scale, patients were asked to rate their pain on a scale from 0 to 10 , where 0 represents "no pain" and 10 represents the "worst possible pain." The error rate of this scale is approximately $2 \%$. The NRS is often used in studies of pain, e.g. by Sadoughi and Akkashe (24), and Jensen and Karoly (25). There is much evidence supporting the NRS validity for pain measurement, regardless of the type of pain and diversity of the population (2426). Moreover, the content analysis was used to evaluate the scientific validity of the demographic questionnaire. To this end, the researcher prepared the initial questionnaire by deciding on the research items and conducting a literature review. Then, the opinions of 10 faculty members of the Nursing and Midwifery Faculty of Ahvaz University of Medical Sciences were obtained and used to introduce the required modifications for developing the final version of the questionnaire.

For observing the ethics of research, an ethical approval was obtained with the number of IR.AJUMS.REC.1396.144 from the University and a permission from the heads and officials of Ahvaz Golestan Hospital. After expressing the study objectives to the subjects, they were ensured that the confidentiality of their personal information would be maintained. The ethical principles of existing resources were also respected. 
The two groups received the routine care according to a cardiologist's order.

In addition to the routine care, the intervention group received local thermotherapy with hot packs warmed to $50^{\circ} \mathrm{C}$ one hour after admission to the Heart Unit, following the administration of NRS. The 20-minute-length intervention was applied to the posterior chest by a nurse once a day. Thermotherapy was administered for five days, during which analgesics were given to patients in case of severe pain according to the specialist's order. Before the intervention, the pain score was recorded in case of the occurrence of heart pain. In addition, the pain score was recorded during the intervention period (five days) until 12 hours after the last session.

The NRS was also administered to the control group immediately after the expression of pain before and after the routine care. It is worth noting that the hot pack temperature was adjusted to $50^{\circ} \mathrm{C}$. Since studies have shown the uselessness of local thermotherapy at a temperature of lower than $45^{\circ} \mathrm{C}$, which could be reached after 23 minutes, thermotherapy sessions in the current study lasted for 20 minutes (27).

The mean and standard deviation were used for describing quantitative variables, and frequency and percentage for qualitative ones. First, the relationship between each variable and the pain intensity variable was separately evaluated after the intervention. Then, variables with $\mathrm{P}<0.2$ were introduced to the multivariate model. Regarding the significant intergroup difference in the pain intensity before the intervention, the variables group therapy and pain intensity could not be included before the intervention. Therefore, the patients were categorized into two groups based on the mean pain intensity, and the univariate and multivariate analyses were conducted separately in these two subgroups. The independent $t$-test, paired $t$-test, Chi-square test, and Pearson correlation coefficient were used for univariate analysis and the multiple linear regression was used for multivariate analysis. All analyses were conducted in SPSS 20.

\section{Results}

76 patients were included in the study. The mean age of the intervention group was $57.59 \pm 10.61$ and 24 (61.5\%) patients were male. Independent $t$-test before the intervention showed a significant difference in the mean pain severity between the intervention and control groups ( $P$ 0.001). However, there was no significant difference in the Chi-square test between the groups in terms of gender, diabetes mellitus, hypertension, and hyperlipidemia $(\mathrm{P}>$ 0.05) (Table 1).
This study showed that the mean pain scores in the intervention group were $3.22 \pm 0.86$ before the intervention and $2.61 \pm 0.7$ after it. In the control group, the mean pain scores were $4 \pm 0.79$ before the intervention and 3.05 \pm 0.66 after it. The results indicated a significant difference in the pain score before and after the intervention ( $P$ $<0.001$ ). According to Table 2, the intergroup comparison also showed a significant difference before the intervention $(\mathrm{P}<0.001)$ and after the intervention $\mathrm{P}=0.005)$.

Using paired $t$-test, there was no significant difference between the two intervention and control groups before and after the intervention with respect to the median pain score of 3.6 ( $\mathrm{P}>0.05)$. However, the mean pain severity in each group alone with respect to the median of 3.6 was accompanied by a significant decrease in pain severity $(\mathrm{P}<$ 0.001) (Table 3 ).

The Pearson correlation coefficient showed no significant relationship between the pain score and gender $(\mathrm{P}=$ $0.103)$, age $(P=0.422)$, high/low blood pressure $(P=0.476)$, diabetes $(P=0.470)$, and hyperlipidemia $(P=0.954)$. The application of the logistic regression to participants with the pain score of lower than 3.6 before the intervention showed that the post-intervention pain score was higher among women than among men, considering the research group as the control variable $(\mathrm{P}=0.039)$. By considering gender as a control variable, there was no intergroup difference before and after the intervention $(P=0.906)$. By considering the research group as the control variable, there was no difference between men and women in terms of the post-intervention severity of pain among those with the pain score of higher than 3.6 before the intervention $(\mathrm{P}=0.786)$. By considering gender as the control variable, there was no intergroup difference in terms of the severity of pain after the intervention $(\mathrm{P}=0.906)$.

In subjects who had a pain severity score of more than 3.6 before the intervention, with control of the variables in the treatment groups, women and men had no significant difference in the pain severity after the intervention ( $\mathrm{P}=$ 0.786). By controlling the variable gender, the two intervention and control groups did not differ in the pain severity after the intervention $(\mathrm{P}=0.25)$.

\section{Discussion}

The results of the current study showed the effectiveness of local thermotherapy in reducing the severity of pain in patients with ACS; however, this reduction was not statistically significant. Regarding the intergroup difference in terms of the severity of pain before the intervention and considering a median pain score in this study, this difference was not significant either before or after the intervention. Nevertheless, the intragroup comparison in- 


\begin{tabular}{|c|c|c|c|}
\hline Variables & Intervention, $\mathrm{N}=39$ & Control, $\mathbf{N}=39$ & Significance Level $^{\text {b }}$ \\
\hline Gender & & & 0.255 \\
\hline Male & $24(61.5)$ & $19(48.7)$ & \\
\hline Female & $15(38.5)$ & $20(51.3)$ & \\
\hline Diabetes & & & 0.354 \\
\hline Yes & $20(51.3)$ & $16(41)$ & \\
\hline No & $19(48.7)$ & $23(59)$ & \\
\hline Blood pressure, mg/kg & & & 0.651 \\
\hline Yes & $21(53.8)$ & $19(48.7)$ & \\
\hline No & $18(46.2)$ & $20(51.3)$ & \\
\hline Hyperlipidemia, mg/kg & & & 0.224 \\
\hline Yes & $5(12.8)$ & $6(15.4)$ & \\
\hline No & $34(87.2)$ & $33(84.6)$ & \\
\hline Age, $y$ & $57.59 \pm 10.61$ & $54.97 \pm 11.41$ & 0.298 \\
\hline Pain intensity & $3.22 \pm 0.86$ & $4 \pm 0.79$ & $<0.001^{\mathrm{c}}$ \\
\hline \multicolumn{4}{|c|}{$\begin{array}{l}\text { a } \text { Quantitative variables are presented as No. (\%) and qu } \\
\text { b Based on chi-square and independent } t \text {-test. } \\
{ }^{c} \text { Significance level at } \mathrm{P}=0.001 \text {. }\end{array}$} \\
\hline Groups Pain Score & Intervention, $\mathrm{N}=39$ & Control, $\mathbf{N}=\mathbf{3 9}$ & Significance Level $^{\text {b }}$ \\
\hline Before intervention & $4 \pm 0.79$ & $3.22 \pm 0.86$ & $<0.001^{\mathrm{C}}$ \\
\hline After intervention & $3.05 \pm 0.66$ & $2.61 \pm 0.7$ & $0.005^{d}$ \\
\hline Significance level & $<0.001^{\mathrm{d}}$ & $<0.001^{\mathrm{d}}$ & \\
\hline \multicolumn{4}{|c|}{$\begin{array}{l}\text { a Values are expressed as mean } \pm \text { SD. } \\
\text { b Based on paired } t \text {-test. } \\
{ }^{c} \text { Significance level at } \mathrm{P}=0.001 . \\
{ }^{\mathrm{d}} \text { Significance level at } \mathrm{P}=0.05 .\end{array}$} \\
\hline Group & Before Intervention & After Intervention & Significance Level $^{\mathbf{b}}$ \\
\hline \multicolumn{4}{|l|}{ Median pain score $<3.6$} \\
\hline Control & $3.03 \pm 0.28$ & $2.41 \pm 0.42$ & $<0.001^{\mathrm{C}}$ \\
\hline Intervention & $2.92 \pm 0.66$ & $2.36 \pm 0.52$ & $<0.001^{\mathrm{c}}$ \\
\hline Significance level & 0.42 & 0.4751 & \\
\hline \multicolumn{4}{|l|}{ Median pain score $>3.6$} \\
\hline Control & $4.43 \pm 0.51$ & $3.33 \pm 0.53$ & $<0.001^{\mathrm{c}}$ \\
\hline Intervention & $4.40 \pm 0.41$ & $3.57 \pm 0.38$ & $<0.001^{\mathrm{c}}$ \\
\hline Significance level & 0.242 & 0.882 & \\
\hline
\end{tabular}

${ }^{\mathrm{a}}$ Values are expressed as mean $\pm \mathrm{SD}$.

${ }^{\mathrm{b}}$ Based on paired $t$-test.

${ }^{\mathrm{c}}$ Significance level at $\mathrm{P}=0.001$.

dicated a significant reduction in the pain score after the intervention. In a study conducted by Haghighatian et al. although the severity of pain in patients with back pain was not significant before the intervention, the pain reduction was greater in the thermotherapy group than in the control group after the intervention (15). Moreover, Yildirim et al. showed the greater effectiveness of local thermotherapy, using hot packs, in reducing the severity of pain in patients with osteoarthritis compared to controls (21). Behmanesh et al. showed a greater reduction in the length of the third stage of labor and the severity of pain among women receiving local thermotherapy compared to a control group (22). Kuwahata et al. showed the effectiveness of sauna therapy in reducing the cardiac size and severity of pain in patients with heart failure (28). Mohammadian et al. also showed the effectiveness of thermother- 
apy in reducing the severity and frequency of pain in the case group that, in turn, reduced the need for opioid medications (5). French et al. showed that local thermotherapy reduced the severity of pain in patients with back pain after five days (29). Michlovitz et al. showed the greater effectiveness of local thermotherapy in relieving pain and reducing joint stiffness in patients with carpal tunnel syndrome (30). The results showed a consistency between the mentioned studies and the current study in terms of the intergroup difference in the severity of pain before the intervention; however, there was inconsistency in terms of thermotherapy effect after the intervention. The following may be the causes of this inconsistency: (I) Providing the control group with local thermotherapy by outsiders, such as family companions, beyond the research period, (II) inappropriate distribution of participants in the intervention and control groups, (III) not investigating the groups in terms of analgesic administration and its repetition, and (IV) differences in the place of hot pack application. Similar to the current study, the hot pack was placed on the posterior chest in the Mohammadian et al. study (5). On the other hand, Jiara, Omama, and Miamuto placed the hot pack on the anterior chest, which could not affect the heart rate (31-33). The effectiveness of local thermotherapy in improving pain in patients with ACS can be attributed to the improved myocardial perfusion as the main cause of pain is the reduced myocardial perfusion. In fact, pain mediators, such as bradykinin and histamine metabolites, are removed from the affected site, thereby preventing the occurrence of pain, providing relief, and reducing cardiac pain. Moreover, the stimulation of thermal receptors increases the endorphin secretion by the descending pain control system that, in turn, relieves the pain. On the other hand, it causes endothelial duplication and increased nitric oxide secretion, thereby improving myocardial perfusion that leads to pain reduction $(14,34)$. The current study did not show any significant relationship between the severity of pain and hyperlipidemia, low/high blood pressure, gender, age, and diabetes; whereas, Imamura et al. reported a reduction in glucose level, blood pressure, hyperlipidemia, and weight, as risk factors for atherosclerosis, through the repetition of thermotherapy for two consecutive weeks (31). This inconsistency with the current study may be due to the difference in the thermotherapy period, which was five days in this study versus two consecutive weeks in Imamura et al study, who reported a significant relationship between the severity of pain and the aforementioned factors after two weeks (31). Mohammadpur also reported a significant relationship between reduced blood pressure and the effect of local thermotherapy (7). Among the research limitations was the use of analgesics for pain management, which was out of the re- searcher's control.

\subsection{Conclusions}

The application of local thermotherapy to the chest of patients with ACS was accompanied by the reduced severity of pain; however, this reduction was not significant. It is recommended to conduct more studies with a greater sample size and placement of hot pack on both anterior and posterior chest for pain reduction.

\section{Acknowledgments}

This paper is the result of a thesis approved by Ahvaz Jundishapur University of Medical Sciences, No. B-9624, which was supported by the vice-chancellor for Research and Technology of Ahvaz Jundishapur University of Medical Sciences. Hereby, the researcher thanks the authorities and staff of Ahvaz Golestan Hospital's ICU, participating patients, and all of the other contributors.

\section{Footnotes}

Authors' Contribution: Study concept and design: Amin Moradkhani, Shahram Baraz, Habib Haybar, Saeed Hesam, and Akram Hematipour; analysis and interpretation of data: Saeed Hesam and Shahram Baraz; drafting of the manuscript: Shahram Baraz, Amin Moradkhani, Akram Hematipour, and Habib Haybar; critical revision of the manuscript for important intellectual content: Shahram Baraz, Saeed Hesam, Akram Hematipour, Amin Moradkhani, and Habib Haybar.

Financial Disclosure: We have no financial interests related to the material in the manuscript.

Funding/Support: This study was supported in part by Ahvaz Jundishapur University of Medical Sciences.

\section{References}

1. Beyranvand MR, Kolahi AA, Ghafelehbashi SHR. Charactristics and final diagnosis of patients with primary diagnosis of acute coronary syndrome. J Babol Univ Med Sci. 2008;10(3):76-82.

2. Thygesen K, Alpert JS, Jaffe AS, Simoons ML, Chaitman BR, White HD, et al. Third universal definition of myocardial infarction. Eur Heart J. 2012;33(20):2551-67. doi: 10.1093/eurheartj/ehs184. [PubMed: 22922414].

3. Shad B, Mirblok F, Goodwill C, Rahmani S, Ghanbari A. [Correlation of cardiovascular blood flow with clinical outcomes in patients with acute coronary syndrome].J Guilan Univ Med Sci. 2016;24. Persian.

4. Sanchis-Gomar F, Perez-Quilis C, Leischik R, Lucia A. Epidemiology of coronary heart disease and acute coronary syndrome. Ann Transl Med.2016;4(13):256. doi: 10.21037/atm.2016.06.33.[PubMed: 27500157]. [PubMed Central: PMC4958723]. 
5. Mohammadian B, Mohammadpour A, Nematollahi MR, Jamiati E. [The effects of local heat therapy in the posterior part of chest on physiologic parameters in the patients with acute coronary syndrome: A randomized double-blind placebo-controlled clinical trial]. Sci J Kurdistan Univ Med Sci. 2017;22(1):72-81. Persian.

6. Dracup K, McKinley S, Riegel B, Moser DK, Meischke H, Doering LV, et al. A randomized clinical trial to reduce patient prehospital delay to treatment in acute coronary syndrome. Circ Cardiovasc Qual Outcomes. 2009;2(6):524-32. doi: 10.1161/CIRCOUTCOMES.109.852608. [PubMed: 20031889]. [PubMed Central: PMC2802063].

7. Mohammadpur A, Mohammadian B, Basiri Moghadam M, Nematollahi MR. The effect of local heat therapy on physiologic parameters of patients with acute coronary syndrome: A randomized controlled clinical trial. Iran J Critical Care Nurs. 2014;7(2):74-83.

8. Lane RD, Reis HT, Hsu CH, Kern KB, Couderc JP, Moss AJ, et al. Abnormal repolarization duration during everyday emotional arousal in long QT syndrome and coronary artery disease. Am J Med.2018;131(5):565572 e2. doi: 10.1016/j.amjmed.2017.12.017. [PubMed: 29309742].

9. Wang AT, Sundt III TM, Cutshall SM, Bauer BA. Massage therapy after cardiac surgery. Seminars in Thoracic and Cardiovascular Surgery. Elsevier; 2010. p. 225-9.

10. Aziznejad Roshan P, Alhani F, Mohammadi E. [Challenges and practical solutions for pain management nursing in pediatric wards]. J Babol Univ Med Sci. 2015;17(12):57-64. Persian.

11. Brennan F, Carr DB, Cousins M. Pain management: A fundamental human right. Anesth Analg. 2007;105(1):205-21. doi: 10.1213/01.ane.0000268145.52345.55. [PubMed:17578977]

12. Ghorbani Moghaddam Z, Jahanpour F, Hajivandi A. [Knowledge attitude and practice of nursing regarding post operative pain management in hospitals affiliated to Bushehr University of Medical Sciences Management]. Nurs J Vulnerable. 2015;1(1):32-41. Persian.

13. Heidari D, Najjar L, Stadiy Z. [The role of nurses in the management of pain in patients with coronary artery disease in Vasei Hospital in Sabzevar]. J Gorgan Univ Med Sci. 2008;10(2):59-64. Persian.

14. Gale GD, Rothbart PJ, Li Y. Infrared therapy for chronic low back pain: A randomized, controlled trial.Pain Res Manag. 2006;11(3):193-6. [PubMed: 16960636]. [PubMed Central: PMC2539004].

15. Haghighatian M, Haghighat F, Rostami Z. [Uses of heat wrap therapy for the relief of patients acute low back pain]. Health Sys Res. 2014;10(2):354-61. Persian.

16. Cameron M. Physical agents in rehabilitation: From research to practice. Philadelphia: WB Saunders company; 1999. p.138-48.

17. Nadler SF, Steiner DJ, Erasala GN, Hengehold DA, Abeln SB, Weingand KW. Continuous low-level heatwrap therapy for treating acute nonspecific low back pain. Arch Phys Med Rehabil. 2003;84(3):329-34. doi: 10.1053/apmr.2003.50102. [PubMed: 12638099].

18. Yaghobi M, Fathi M, Roshani D, Valiee S, Moradi M, Hasnghani H. [Comparison of the effect of infra-red and hot pack on reduction of pain associated with lumbar discopathy]. Sci J Kurdistan Univ Med Sci. 2012;17(2):72-81. Persian.

19. Uchiyama-Tanaka Y. Effect of thermal therapy using hot water bottles on brain natriuretic peptide in chronic hemodialysis patients. Cardiol Ther. 2012;1(1):2. doi: 10.1007/s40119-012-0002-z. [PubMed: 25135156] [PubMed Central: PMC4107443].

20. Takayama S, Seki T, Watanabe M, Takashima S, Sugita N, Konno S, et al Changes of blood flow volume in the superior mesenteric artery and brachial artery with abdominal thermal stimulation. Eur J Integr Med. 2009;1(4):234. doi: 10.1016/j.eujim.2009.08.022.

21. Yildirim N, Filiz Ulusoy M, Bodur $H$. The effect of heat application on pain, stiffness, physical function and quality of life in patients with knee osteoarthritis. J Clin Nurs. 2010;19(7-8):1113-20. doi: 10.1111/j.13652702.2009.03070.x. [PubMed: 20492056].

22. Behmanesh F, Pasha $H$, Zeynalzadeh M. [Effect of heat therapy on pain severity and the end of labor in primigravida women]. Bimonthly JHormozgan Univ Med Sci. 2009;12(4):261-9. Persian.

23. Cui J, Sinoway LI. Cardiovascular responses to heat stress in chronic heart failure. Curr Heart Fail Rep. 2014;11(2):139-45. doi: 10.1007/s11897014-0191-y. [PubMed: 24599558]. [PubMed Central: PMC4042428].

24. Sadoughi M, Akkashe G. [Effectiveness of cognitive-behavioral therapy on reduction of chronic tension headache]. J Shahrekord Uuniv Med Sci. 2009;11(3):85-92. Persian.

25. Jensen MP, Karoly P. Self-report scales and procedures for assessing pain in adults. In: Turk DC, Melzack R, editors. Handbook of pain assessment. New York, NY, US: Guilford Press;1992. p. 135-51.

26. Turk DC, Melzack R. Handbook of pain assessment. Guilford Press; 2011.

27. Knight K, Knight KL, Draper DO. Therapeutic modalities: The art and science. Lippincott Williams \& Wilkins; 2012.

28. Kuwahata S, Miyata M, Fujita S, Kubozono T, Shinsato T, Ikeda Y, et al. Improvement of autonomic nervous activity by Waon therapy in patients with chronic heart failure. J Cardiol. 2011;57(1):100-6. doi: 10.1016/j.jjcc.2010.08.005. [PubMed: 20884178].

29. French SD, Cameron M, Walker BF, Reggars JW, Esterman AJ. Superficial heat or cold for low back pain. Cochrane Database Syst Rev. 2006;(1).CD004750. doi:10.1002/14651858.CD004750.pub2. [PubMed: 16437495].

30. Michlovitz S, Hun L, Erasala GN, Hengehold DA, Weingand KW. Continuous low-level heat wrap therapy is effective for treating wrist pain. Arch Phys Med Rehabil. 2004;85(9):1409-16. [PubMed: 15375809].

31. Imamura M, Biro S, Kihara T, Yoshifuku S, Takasaki K, Otsuji Y, et al. Repeated thermal therapy improves impaired vascular endothelial function in patients with coronary risk factors. J Am Coll Cardiol. 2001;38(4):1083-8. [PubMed: 11583886].

32. Kihara T, Biro S, Imamura M, Yoshifuku S, Takasaki K, Ikeda Y, et al Repeated sauna treatment improves vascular endothelial and cardiac function in patients with chronic heart failure. J Am Coll Cardiol. 2002;39(5):754-9. [PubMed: 11869837].

33. Miyamoto H, Kai H, Nakaura H, Osada K, Mizuta Y, Matsumoto A, et al Safety and efficacy of repeated sauna bathing in patients with chronic systolic heart failure: A preliminary report.J Card Fail. 2005;11(6):4326. doi: 10.1016/j.cardfail.2005.03.004. [PubMed: 16105634].

34. Cameron MH. Physical agents in rehabilitation: From research to practice. Philadelphia: WB Saunders Company;1999. p. 138-48. 